\title{
Anisotropic third-order magneto-optical Kerr effect
}

\author{
A. V. Petukhov ${ }^{\mathrm{a})}$ and Th. Rasing \\ Research Institute for Materials, 6525 ED Nijmegen, The Netherlands \\ T. Katayama \\ Electrotechnical Lab. Tsukuba-shi, Ibaraki 305, Japan \\ N. Nakajima \\ Photon Factory, National Laboratory, High Energy Physics, Tsukuba-shi, Ibaraki 305, Japan \\ Y. Suzuki \\ JRCAT-NAIR, Tsukuba-shi, Ibaraki 305, Japan
}

The magnitude of the polar magneto-optical Kerr effect in reflection from thick iron epitaxial films is experimentally found to strongly depend on the crystallographic orientation of the film, with variations of more than $20 \%$. Similar results are obtained on the (001) and (110) faces of a bulk Fe crystal. It is shown that this anisotropy can be described as a third-order effect in the magnetization $\mathbf{M}$ on the optical response $\epsilon(\omega, \mathbf{M})$. Our analysis can also be used to understand the transversal Kerr effect at normal incidence recently observed by Gridnev et al. (C) 1998 American Institute of Physics. [S0021-8979(98)46611-8]

Magneto-optics provides a simple and quite universal way to probe the magnetization $\mathbf{M}$ of a ferromagnetic medium. ${ }^{1-4}$ Most of the magneto-optical phenomena arise due to the effect of $\mathbf{M}$ on the dielectric constant of a medium $\epsilon(\mathbf{M})$. Assuming that this effect is weak, the $\epsilon(\mathbf{M})$ dependence is often expanded in powers of $\mathbf{M}$ yielding ${ }^{5}$

$\stackrel{\leftrightarrow}{\epsilon}(\mathbf{M})=\overleftrightarrow{\epsilon}^{(0)}+4 \pi i f \cdot \mathbf{M}+4 \pi \mathrm{g}: \mathbf{M M}+4 \pi i \mathrm{~h}: \mathbf{M M M}+\cdots$,

where $\epsilon^{(0)}$ denotes the magnetization-independent part of $\epsilon(\mathbf{M})$ and the tensors $\mathrm{f}, \mathrm{g}$, and $\mathrm{h}$ describe the linear-, second-, and third-order magnetization-induced corrections to the dielectric function of the magnetic medium. So far the main attention has been paid to the magneto-optical effects arising via the second and third term in Eq. (1). For instance, to describe magnetic circular dichroism (MCD) it is usually enough to account for the term $4 \pi i f \times \mathbf{M}$ that is linear in the magnetization. Upon light reflection, MCD results in the magneto-optical Kerr effect (MOKE) which is used to study magnetism in opaque media. ${ }^{1}$ The second-order magnetization-induced term $4 \pi \mathrm{g}$ : $\mathbf{M M}$ results in the linear birefringence. ${ }^{2}$ Higher order terms in $\mathbf{M}$ in Eq. (1) are usually neglected. However, they may result in new magnetooptical effects and thus become important. In particular, a third-order contribution $(\propto h)$ to Eq. (1) has been recently observed by Gridnev et al. ${ }^{6}$ to contribute to the transversal magneto-optical Kerr effect upon light reflection from the (111) face of $\mathrm{LiFe}_{5} \mathrm{O}_{8}$.

In this work we analyze novel magneto-optical effects that can originate via higher-order (and anisotropic) contributions in the magnetization dependence of the dielectric response in cubic media. In particular, these contributions can describe experimentally observed anisotropy of the magneto-optical Kerr rotation in reflection from (001) and

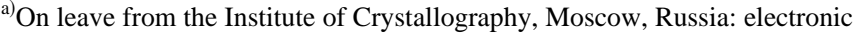
mail: petukhov@sci.kun.ni
(110) oriented iron epitaxial films and (001) and (110) faces of a bulk Fe crystal. A similar effect has also been observed on epitaxial hcp Co films, ${ }^{3}$ where the anisotropy is linear in the magnetization. ${ }^{3,4}$ For cubic bcc iron, however, this linear effect is forbidden by symmetry and the anisotropy can be understood as a third-order effect of the magnetization. We also demonstrate how the orientation dependence of the polar MOKE is related to the transversal third-order MOKE observed in Ref. 6.

The epitaxial $\mathrm{Fe}(001)$ and (110) films with the thickness 700-1000 $\AA$ were grown on $\mathrm{Au}(001)$ and $\mathrm{Au}(111)$ buffer layers as described in Ref. 7. The bulk samples were prepared by cutting an iron single crystal along (001) and (110) planes with subsequent polishing by a diamond paste. The MOKE spectra of epitaxial Fe films were measured in the range of 4-10 eV using the synchrotron facility described in Ref. 8. Similar measurements in the range $1.55-6 \mathrm{eV}$ on epitaxial films and bulk samples were done on a Kerr spectrometer.

In Fig. 1 we show the spectra of the Kerr rotation angle $\phi_{K}$ and the Kerr ellipticity $\eta_{K}$ measured on thick Fe epitaxial films with the (001) and (110) orientation. A strong anisotropy $\Delta \phi_{K}=\phi_{K}^{(001)}-\phi_{K}^{(110)}$ and $\Delta \eta_{K}=\eta_{K}^{(001)}-\eta_{K}^{(110)}$ is
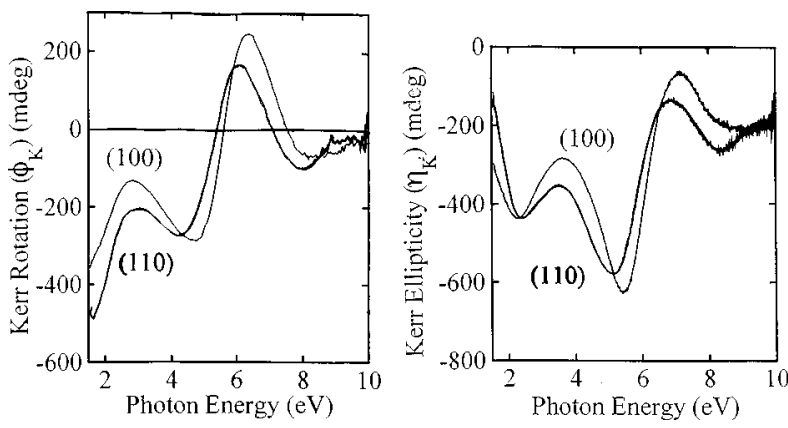

FIG. 1. The Kerr rotation (left panel) and Kerr ellipticity (right panel) spectra measured on epitaxial $\mathrm{Fe}(001)$ and $\mathrm{Fe}(110)$ films. 

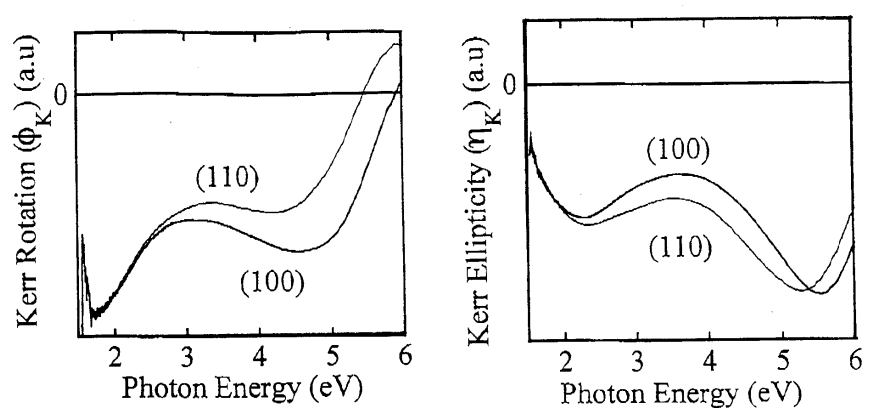

FIG. 2. The same as in Fig. 1 but measured on two faces of a bulk iron crystal.

clearly seen. For the thick Fe films the effect of quantum well states should be negligible and the natural explanation of this anisotropy could then be a difference in the structure of the two studied films. For instance, a different strain of the Fe lattice may affect the magneto-optical parameters. Alternatively, one can think about a different roughness of the different film surfaces as a possible source of the orientation dependence of MOKE.

However, an additional study performed on different faces of iron bulk single crystals contradicts this simple explanation. In Fig. 2 we show the MOKE spectra measured upon light reflection from $\mathrm{Fe}(001)$ and $\mathrm{Fe}(110)$. In this case the effect of the lattice strain should be much smaller, if not negligible, in comparison to that expected in epitaxial films. The surface (micro-) roughness of the faces of the bulk crystal, which can result from the sample polishing, is obviously expected to be distinctly different from the surface (micro-) roughness of epitaxial films which can arise during the film growth. However, the great similarity between the results presented in Figs. 1 and 2 is obvious. Only at small photon energies $\hbar \omega \leqslant 2 \mathrm{eV}$ there is a clear anisotropy found for the epitaxial films while the single crystal faces do not show this. This observation strongly suggests that the MOKE anisotropy at $\hbar \omega>2 \mathrm{eV}$ is an intrinsic effect of a perfect Fe crystal rather than related to the mechanisms described above.

For cubic media the first two tensors $\overleftrightarrow{\epsilon}^{(0)}$ and $f$ in the expansion (1) reduce to scalars $\epsilon_{0}$ and $f_{0}$ times the unity symmetric and antisymmetric tensors, respectively. Therefore, up to the term linear in the magnetization, the relation between the induction $\mathbf{D}(\omega)$ and the electric field $\mathbf{E}(\omega)$ of the light wave can be written in a vector form:

$$
\mathbf{D}=\epsilon_{0} \mathbf{E}+4 \pi i f_{0} \mathbf{E} \times \mathbf{M} .
$$

Although MCD depends on the mutual orientation of the light propagation direction and the magnetization $\mathbf{M}$, within the approximation of Eq. (2) MCD is isotropic in a sense that it does not depend on the orientation of $\mathbf{E}$ and $\mathbf{M}$ relative to the crystallographic axes. Therefore, to describe the anisotropic MCD effect in a cubic medium one has to account for the higher-order terms in the expansion (1), which are anisotropic even in high-symmetry cubic media. The second-order term $4 \pi g_{i j k l} M_{k} M_{l}$ is even in the magnetization and therefore cannot contribute to MCD. In order to account for the anisotropy of MCD one therefore has to consider the thirdorder term.
In general, an axial fifth-rank tensor in a cubic medium has ten independent nonvanishing elements. ${ }^{9}$ The permutation symmetry of $h_{i j k l m}$ with respect to the last three indices and the Onsager symmetry $h_{i j k l m}=-h_{j i k l m}$ reduces this number to only 2 . In the following it is convenient to introduce a contracted notation. We define a ten-dimensional vector $\overrightarrow{\mathbf{M M M}}$ with components

$$
\left.\overrightarrow{\mathbf{M M M}}=\mid \begin{array}{c}
M_{1}^{3} \\
M_{2}^{3} \\
M_{3}^{3} \\
3 M_{2} M_{3}^{2} \\
3 M_{3} M_{1}^{2} \\
3 M_{1} M_{2}^{2} \\
3 M_{3} M_{2}^{2} \\
3 M_{1} M_{3}^{2} \\
3 M_{2} M_{1}^{2} \\
6 M_{1} M_{2} M_{3}
\end{array}\right),
$$

where $M_{1}, M_{2}$, and $M_{3}$ denote the projections of $\mathbf{M}$ on the principal crystallographic axes [100], [010], and [001]. The axial fifth-rank tensor $\mathrm{h}$ can then be written as a third-rank tensor $\kappa_{i j K}$ where the last index $K$ runs from 1 to 10 . The nonvanishing elements of $\kappa_{i j K}$ are

$$
\begin{gathered}
\kappa_{1} \equiv \kappa_{123}=\kappa_{231}=\kappa_{312}=-\kappa_{132}=-\kappa_{213}=-\kappa_{321}, \\
\kappa_{2} \equiv \kappa_{127}=\kappa_{238}=\kappa_{319}=-\kappa_{139}=-\kappa_{328}=-\kappa_{217} \\
=\kappa_{125}=\kappa_{236}=\kappa_{314}=-\kappa_{134}=-\kappa_{326}=-\kappa_{215} .
\end{gathered}
$$

For isotropic media the same elements of $\kappa_{i j K}$ are nonvanishing but the number of independent elements is reduced to one because of an extra relation $\kappa_{1}=3 \kappa_{2}$ between the $\kappa_{1}$ and $\kappa_{2}$ elements. In cubic media the anisotropic element $\kappa_{\text {an }}$ $=\kappa_{1} / 3-\kappa_{2} \neq 0$ leads to the anisotropy in MCD.

Now we introduce the laboratory coordinate system $(x y z)$, where the $z$ axis is normal to the surface and along the magnetization vector $\mathbf{M}=\mathbf{e}_{z} M$. The transformation matrix A from the crystallographic (123) to the laboratory ( $x y z)$ frame is taken in the form

$$
A=\left(\begin{array}{ccc}
\cos \theta \sin \psi & \cos \theta \cos \psi & -\sin \theta \\
-\cos \psi & \sin \psi & 0 \\
\sin \theta \sin \psi & \sin \theta \cos \psi & \cos \theta
\end{array}\right),
$$

where the angles $\theta$ and $\psi$ determine the orientation of the surface normal $\mathbf{e}_{z}$ with respect to the crystallographic axes. Using Eqs. (4) and (5) one can find the $x y$ element of $\overleftrightarrow{\epsilon}\left(\mathbf{e}_{z} M\right)$ :

$$
\begin{aligned}
\boldsymbol{\epsilon}_{x y}\left(\mathbf{e}_{z} M\right)= & 4 \pi i\left[f_{0} M+\kappa_{1} M^{3}-6 \kappa_{\mathrm{an}} \sin ^{2} \theta\left(\cos ^{2} \theta\right.\right. \\
& \left.\left.+\sin ^{2} \theta \cos ^{2} \psi \sin ^{2} \psi\right) M^{3}\right]
\end{aligned}
$$

that determines the MCD for light propagating along $z$. The last term in Eq. (6) is anisotropic, i.e., it depends on the orientation of the surface relative to the cubic frame. For the (001) face $\theta=0$ and Eq. (6) yields

$$
\boldsymbol{\epsilon}_{x y}^{(001)}\left(\mathbf{e}_{z} M\right)=4 \pi i\left[f_{0} M+\kappa_{1} M^{3}\right]
$$


while for the (110) face $\theta=\pi / 4, \psi=0$ and

$$
\epsilon_{x y}^{(110)}\left(\mathbf{e}_{z} M\right)=4 \pi i\left[f_{0} M+\kappa_{1} M^{3}-\frac{3}{2} \kappa_{\mathrm{an}} M^{3}\right] .
$$

Obviously, $\epsilon_{x y}^{(001)} \neq \epsilon_{x y}^{(110)}$ and the third-order term in expansion (1) can describe the observed anisotropy.

The analysis above can also be used to describe the observed third-order transversal $(\mathbf{k} \perp \mathbf{M}$, where $\mathbf{k}$ denotes the light wave vector) Kerr effect reported in Ref. 6. We take the light incident along the surface normal $z$ and the magnetization parallel to the surface $\mathbf{M}=\mathbf{e}_{x} M$. In cubic media the linear in the magnetization contribution $i f_{0} \mathbf{E} \times \mathbf{M}$ to the induced polarization is then always along $\mathbf{k}$ and, therefore, does not radiate into the direction of light propagation. The second-order contribution $\mathbf{g : M M}$ is reciprocal and cannot contribute to the MCD and one again arrives to the thirdorder term which gives rise to the transversal MCD via

$$
\epsilon_{x y}\left(\mathbf{e}_{x} M\right)=h_{x y x x x} M^{3},
$$

where $h_{x y x x x}=\kappa_{\text {an }} f(\theta, \psi, \phi)$ denotes the magnitude of the corresponding tensor element in the laboratory coordinate system which depend on the relative orientation of the two frames given by the Euler angles $\theta, \psi$, and $\phi$, where the additional angle $\phi$ describes the azimuthal orientation of the sample which becomes important for the transversal geometry. We note that this transversal MCD vanishes on the even-fold rotation symmetry (001) and (110) faces of cubic fcc and bcc crystals. Indeed, the rotation of the sample by $\pi$ reverses the direction of $\mathbf{M}=\mathbf{e}_{x} M$ while $\epsilon_{x y}$ and $h_{x y x x x}$ should be unchanged since $(x, y) \rightarrow(-x,-y)$ is a symmetry operation for these faces and $\epsilon_{x y}\left(\mathbf{e}_{x} M\right)$ should therefore vanish for any $\phi$. For other faces the transversal MCD is present. For instance, for the (111) face one can calculate the function $f(\theta, \psi, \phi)$ to obtain

$$
\epsilon_{x y}\left(\mathbf{e}_{x} M\right)=-\frac{4 \pi i}{\sqrt{2}} \kappa_{\mathrm{an}} M^{3} \sin 3 \phi .
$$

Note that this effect is anisotropic during azimuthal rotation of the sample. It vanishes every time when the magnetization is normal to one of the mirror symmetry planes $(01 \overline{1}),(10 \overline{1})$, and (110) of the (111) face. Comparing Eqs. (7), (8), and (10) one can see that a measurement of the transversal MOKE on $\mathrm{Fe}(111)$ similar to that done by Gridnev et al. ${ }^{6}$ can be used for an unambiguous verification of the mechanism of the anisotropy of the polar Kerr effect proposed in this work.

In conclusion, we have observed a strong surface orientation dependence of the magneto-optical Kerr effect in the polar configuration that can be understood as a third-order effect in the magnetization on the optical response. We give a phenomenological description of this effect, which can also explain the transversal Kerr effect at normal incidence on odd-fold symmetry faces of a cubic crystal observed by Gridnev et al.

Fruitful discussions with Roman V. Pisarev and Andrei Kirilyuk are acknowledged. The present work was partially supported by the HCM fellowship ERBCHBICT-941761 and TMR network "NOMOKE.',

${ }^{1}$ U. Gradmann, J. Magn. Magn. Mater. 100, 481 (1991); D. Bader, ibid. 100, 440 (1991).

${ }^{2}$ See, e.g., J. Ferré and G. A. Gehring, Rep. Prog. Phys. 47, 513 (1984).

${ }^{3}$ D. Weller, G. R. Harp, R. F. C. Farrow, A. Cebollada, and J. Sticht, Phys. Rev. Lett. 72, 2097 (1994).

${ }^{4}$ P. M. Oppeneer, T. Kraft, and H. Eschrig, Phys. Rev. B 52, 3577 (1995).

${ }^{5}$ L. D. Landau and E. M. Lifshitz, Electrodynamics of Continuous Media (Pergamon, New York, 1960).

${ }^{6}$ V. N. Gridnev, B. B. Krichevtsov, V. V. Pavlov, and R. V. Pisarev, Pis'ma Zh. Eksp. Teor. Fiz. 65, 65 (1997) [JETP Lett. 65, 68 (1997)].

Note that the magnetoelectric contribution to the optical response, which is also observed in this work, is forbidden in centrosymmetric iron.

${ }^{7}$ T. Katayama, N. Nakajima, N. Okusawa, Y. Miyauchi, T. Koide, T. Shidara, Y. Suzuki, and S. Yuasa, J. Magn. Magn. Mater. (in press).

${ }^{8}$ T. Sugimoto, T. Katayama, Y. Suzuki, T. Koide, T. Shidara, M. Yuri, A. Itoh, and K. Kawanishi, Phys. Rev. B 48, 16432 (1993).

${ }^{9}$ A. V. Petukhov, I. L. Lyubchanskii, and Th. Rasing, Phys. Rev. B 56, 2680 (1997) 